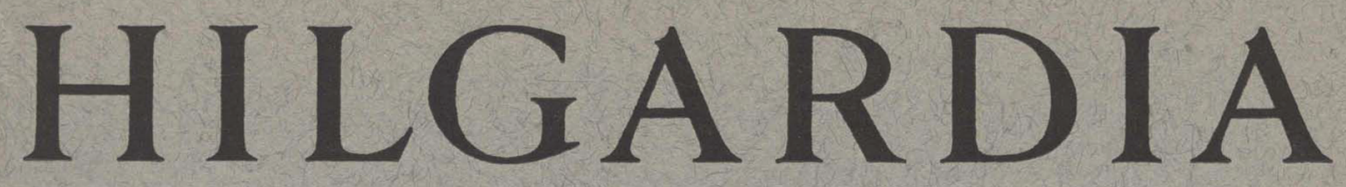

A Journal of Agricultural Science Published by the California Agricultural Experiment Station

\title{
A PROCEDURE FOR DETERMINING RELATIVE DENSITIES OF BROWN ALMOND MITE POPULATIONS ON ALMOND TREES
}

FRANCIS M. SUMMERS and GEORGE A. BAKER 
Brown almond mites reside on woody portions of their fruit tree hosts, from which they make excursions to feed on leaves only during favorable periods of the day. Changes in temperature and possibly in sunlight greatly affect their migrations back and forth on almond trees. Their excursions for food introduce serious errors in sampling whenever attempts are made to compare different populations on a basis of mites counted in samples of plucked leaves.

The method described here avoids this difficulty since the mites are counted in samples of leaves and wood. Mites present on a clump of almond foliage are shaken onto a sheet of paper and permanently fixed in position by crushing. Derivation of numerical data from the patterns of crushed mites and appropriate statistical analyses are included in the discussion. The design indicated as being adequate for testing relative efficiency of treatments requires plots of four or five similar trees. Thirty individual twigs from each plot are usually sufficient. 




A Journal of Agricultural Science Published by

the California Agricultural Experiment Station

VoL. 21

APRIL, 1952

No. 13

\section{A PROCEDURE FOR DETERMINING RELATIVE DENSITIES OF BROWN ALMOND MITE POPULATIONS ON ALMOND TREES ${ }^{1}$}

\author{
FRANCIS M. SUMMERS ${ }^{2}$ and GEORGE A. BAKER ${ }^{3}$
}

\section{INTRODUCTION}

Population densities of the brown almond mite, Bryobia praetiosa Koch (also known as the clover mite), are difficult to estimate on almond trees by methods of sampling based on numbers of mites counted on leaves when the leaves are plucked and examined one at a time. These estimates are usually expressed as numbers of mites per leaf or per sample of leaves. The vagaries of methods using data of this type have contributed materially to existing confusion about the merits of new and old materials used for controlling the pest.

The brown almond mite is a transient leaf-feeding species that shows gregarious tendencies on the bark of its fruit tree hosts. It has alternating phases of residence (rest, torpidity, and reproduction) on the woody parts and of feeding on leaf surfaces that are clearly correlated with environmental fluctuations. Available information further indicates that only a small part of the entire population of active mites visits almond leaves when external conditions are conducive to feeding.

The purpose of this paper is to describe a new procedure for determining relative densities of brown almond mite populations, a procedure that is not appreciably affected by diurnal changes in the distribution of mites on leaves and wood. It has been used in control work for two seasons, with consistent results for evaluating treatments applied to almonds. An infestation of brown almond mites almost runs its course on almond trees before two-spotted or Pacific mites appear in numbers sufficient to complicate matters. It is therefore usually possible to work with almost pure populations of brown almond mites on this host. Whether the procedure can be adapted for use on other types of fruit trees is not yet known. It probably cannot be adapted for webspinning tetranychids or for plants having mixed populations.

The data herein presented were obtained primarily to show the range of

${ }^{1}$ Received for publication December 10, 1951.

${ }^{2}$ Lecturer in Entomology and Assistant Entomologist in the Experiment Station, Davis.

${ }^{3}$ Associate Professor of Mathematics and Associate Statistician in the Experiment Station, Davis. 
reliability of the method now used. Experiments on the control of the pest will be described elsewhere. They are included here only to illustrate test procedures.

\section{ECOLOGICAL BASIS OF THE PROCEDURE}

The causal relations between environmental fluctuations and movements of mites from leaves to wood and vice versa are not well understood at present. By means of certain crude techniques it was found that the feeding population stabilizes on almond leaves during daylight hours within approximate temperature limits of $70^{\circ}$ to $85^{\circ} \mathrm{F}$. Within the range of $80^{\circ}$ to $85^{\circ}$, feeding individuals become sensitive to sunlight and will avoid unshaded leaves. The wood-dwelling phase stabilizes at night or at other times when temperatures are below $60^{\circ}$ or above $95^{\circ}$. It is possible, therefore, to draw from heavily infested almond trees samples of leaves that give erroneous estimates of the size of the actual population. Furthermore, the character of the distribution can change during the interval of time required to make direct field counts of mites on 50 or more leaves so that the first and last sample leaves drawn do not represent the same populations.

In order to make objective comparisons between treatments applied for control, it was desirable to devise a sampling procedure that is not greatly disturbed by shifts of the mite population from resting to feeding phases and that eliminates the necessity of working within prescribed limits of weather conditions.

There are several working hypotheses upon which this sampling procedure is based. One hypothesis concerns the phenomenon of dispersal. Although the movements of mites between leaves and wood may be rapid and frequent, the mites associated with bits of foliage tend to remain localized. The dispersal from infested to uninfested areas is a slow process on mature trees. Isolated shoots borne on old wood of lightly infested trees are heavily damaged before emigration occurs. The nearest uninfested foliage remains relatively clean until midseason. This tendency toward sluggish dispersal is also evident in treated plots. Trees disinfested with sprays applied very early in the season coexist side by side with heavily infested trees for periods of three months or longer without being appreciably reinfested. Similarly, the mites remaining in clumps of infested foliage that are missed during spray operations do not disperse rapidly even after chemical residues become inert. Data sheets obtained from more than 125 treated plots indicate that spray "misses" on trees sprayed in late February are discernible in June.

Distribution of mites. The spatial distribution of mites on host trees appears to be correlated with the surface pattern of bark on young trees. The character of the bark's surface, especially its degree of roughness, in some way determines where the mite population will reside during the phase of repose and reproduction. Young, rapidly growing almonds and peaches are seldom severely damaged by brown mites although they do support small populations of the pest. On the smooth-textured bark of these young trees the mites do not congregate in masses close to the foliage. Most of them tend to distribute themselves throughout the length of a branch or hanger, and some tend to retreat to a main scaffold branch before settling. On the other hand, 
the bark of woody terminals and lateral stems of mature almonds is very convoluted or annulated. On this kind of wood the mites congregate in large numbers at or near the bases of the leafy shoots upon which they feed. The common varieties most susceptible to attack by brown mites are Nonpareil, Texas, and Peerless. The woody framework supporting green shoots on these varieties is rough-textured distally. It is believed that a short section of branch or twig removed from these trees after the leafy shoots have developed will bear not only the mites actually feeding on the leaves at the moment, but also most of the resting mites that normally feed on these leaves. Although the statement cannot be amplified further at this time, the numbers of mites removed from both wood and leaves of small foliage samples are believed to serve as population indices for comparative purposes.

Foliage samples. The sampling process is based on the numbers of mites dislodged from relatively uniform bits of foliage or twigs. Since twigs of nearly uniform length or stem diameter do not bear equal numbers of leaves or shoots, this inequality is adjusted in the ratio mites per shoot, a shoot being the tuft of leaves developed from each bud on the twig.

The choice in size of samples taken in trials to date was arbitrary. Six twigs were cut from the perimeter of each of five trees per plot, or a total of 30 twigs per plot.

Complications introduced by sampling often or periodically are avoided by evaluating treatments on the basis of sets of data taken at one time, close to the peak of the seasonal build-up. Supplementary inspections or samplings are necessary, of course, to establish population trends or peculiarities of treatments. The brown almond mite infestations on almonds in the Sacramento and San Joaquin valleys increase slowly to a maximum in late spring. The crisis of the attack is likely to occur in late May or early June. If average warm weather persists thereafter, the number of mites declines sharply during late June. The pest is of little consequence after mid-July. The decline coincides with the onset of diapause in the midsummer eggs (Summers, 1950)." It is therefore practical to apply sprays whenever necessary and to appraise the results at the climax of the mite attack. In this way variously timed sprays can be evaluated in comparable terms.

Recording of data. The collection of field data is most effectively done by two persons, one to cut the twigs and the other to beat the mites onto record sheets. A team of two persons, working under favorable conditions, can process about 40 twigs per hour.

Twigs having 12 to 16 inches of mature wood are clipped from the outer foliage, at convenient heights and from different positions around the circumference. They are cut midway between nodes, through the longest internode whenever possible. Twigs are selected for approximate uniformity in size and shape but at random in degree of injury by mites. Six twigs are gently pruned from a given tree, and the nuts carefully snipped off. The twigs are then laid side by side in a shallow tray and carried to a convenient place for beating.

The mites are jarred from the twig onto a sheet of scratch paper, preferably ruled, $81 / 2 \times 11$ inches in size. Air currents interfere with this operation. Even

"See "Literature Cited" for complete data on citations, referred to in text by name of author and date of publication. 
a light breeze requires that the jarring be done in a shelter or indoors. In very calm weather it may be done in the open or in the trunk compartment of an automobile.

The shoots on a given twig are counted and the number is written at the top of a record sheet, for instance, 5-3-15-fifth tree, third twig having 15 shoots. This sheet is then placed on a board or a table top and covered with an inverted wire rack or cage that provides an open-mesh grid about 4 inches above the paper. The authors use a $12 \times 16$-inch wire dish-draining rack modified only by the addition of an iron bar against which the twigs are beaten. The bar is a $5 / 16^{-i n c h}$ rod bent $U$-shape and brazed to the side supports of the inverted rack to provide a transverse anvil or striking bar about 8 inches from the table top and somewhat above the middle of the record page. The wire grid above the paper minimizes the slapping or fanning of the record sheet by leaves when the twig is being beaten.

The transfer of mites from twig to paper is quickly done. The operator loosely grasps the twig by its leafy end and sharply taps the butt end against the crossbar. Only two to three sharp taps are required to dislodge most of the individuals resting on the wood. The twig is then inverted and its leafy or terminal end beaten to recover mites from the green foliage. The twig is again inverted and the process repeated. Prolonged beating accomplishes very little aside from breaking up the twig and depositing undesirable trash; eight to ten sharp taps usually suffice. The number of mites remaining on the twig thereafter is believed to be inconsequential.

The grid is then removed and large particles of debris are picked from the page with forceps. The record page containing the mass of crawling mites is then picked up and very gently inclined to the vertical. Dust, egg masses, fine particles of bark and old, spray-killed mites fall from the page, leaving the living mites clinging firmly to the paper. Within this short time interval the crawling mites redistribute themselves into a typical dispersed pattern. The sheet is replaced on the table and a press sheet laid over the record sheet, with mites sandwiched between. The upper paper is then pressed by hand to crush the mites and produce a pattern of spots. Experience has shown that pressing by hand gives a clearer pattern than pressing by roller. Excessive pressure gives an undesirable result. Conversely, inadequately crushed mites drop away without leaving a perceptible blot. The paired "squeeze" sheets, one a duplicate of the other, are stacked for analysis at a later time. Patterns produced by crushed mites persist and are legible for a year or longer.

A few individual mites are lost in each stage of the sampling process yet the masses finally crushed into the record page represent the major fraction of the number originally inhabiting the sample twig.

The numbers of mites recovered from infested twigs are often greater than can be counted without tedious effort. Therefore, a second sampling process is introduced when the dead mites or spots on the squeeze sheets are counted. The distribution pattern of the crushed mites is nonhomogeneous. Typically, the spots are slightly more concentrated near the center of the page and less so toward the margins. The crushed mites are counted on a portion of the page with the aid of a template. The template was made with five apertures, each having an area of one square inch; one aperture was in the center and four 
equally spaced on 2-inch radii with one aperture in each quadrant. The template is dropped onto the squeeze sheet so that the central aperture falls over the population epicenter, if any. The spots are easily counted with a loupe and hand tally register.

Since the counts so obtained represent only a fraction of the original population, the final unit recorded for each twig sample must be expressed as numbers of mites per shoot per template. Examples of data collected from squeeze sheets are shown in table 1 to illustrate the observed range of variation between individual twigs, between trees, and between repeated samplings within a plot.

Plots and treatments. The orchard selected contained 15 rows of 21 trees each. Three varieties were planted in two rows each, two rows of Nonpareil alternating with two rows of either Peerless or NePlus. The trees were 15 years old and of fairly uniform growth within varieties. The infestation of mites was moderately heavy throughout, but levels of damage appeared to be greater at the east end than at the west end of the rows. The plots were arranged as shown in figure 1. Untreated strips of three trees deep were left across the ends and treatments were applied in strips of three trees across varieties in the area between the check strips. All samplings were restricted to Nonpareil trees and to next adjacent twin rows for replicate plots whenever possible.

Sprays were applied with a conventional high pressure sprayer operating at 400 psi. The trees were sprayed for full coverage with a single-nozzle orchard gun. The volumes of spray applied varied between 350 to 400 gallons per 45 trees planted 48 to the acre.

The experiment was planned to differentiate between a treatment having a decided and immediate effect on the mites (TEPP) and a similar treatment modified by the addition of a supplement (TEPP plus sulfur). The additional treatments described are a part of another experiment but they have been included here for use in comparing single samplings.

The materials, amounts per 100 gallons of spray, and plot designations were as follows:

A, B (TEPP) Tetraethyl pyrophosphate, $1 / 2$ pint 20 per cent solution*

C, D (TEPPS) Tetraethyl pyrophosphate, $1 / 2$ pint 20 per cent solution, plus wettable sulfur, 5 pounds

I (K-6451) p-Chlorophenyl p-chlorobenzenesulfonate, 2 pounds of a 50 per cent wettable powder $\dagger$

J (923) 2,4-Dichlorophenyl benzenesulfonate, 11/2 pints of a 50 per cent emulsifiable concentrate**

$\mathrm{K}$ (R-242) p-Chlorophenol phenylsulfone, 3 pounds of a 40 per cent wettable powder $\ddagger$

E, F (Check) Untreated trees, east end of rows

G, H (Check) Untreated trees, west end of rows.

The sprays were applied on May 23, 1951, and the various plots were sampled during the period of June 7 to June 12 .

\footnotetext{
* "Vapotone" (TEPP), California Spray Chemical Corporation.

$\dagger$ "Ovotran" (K-6451), Dow Chemical Company.

* "Genite" (923), General Chemical Division, Allied Chemical and Dye Corporation.

$\ddagger$ "Sulphenone" (R-242), Stauffer Chemical Company.
} 


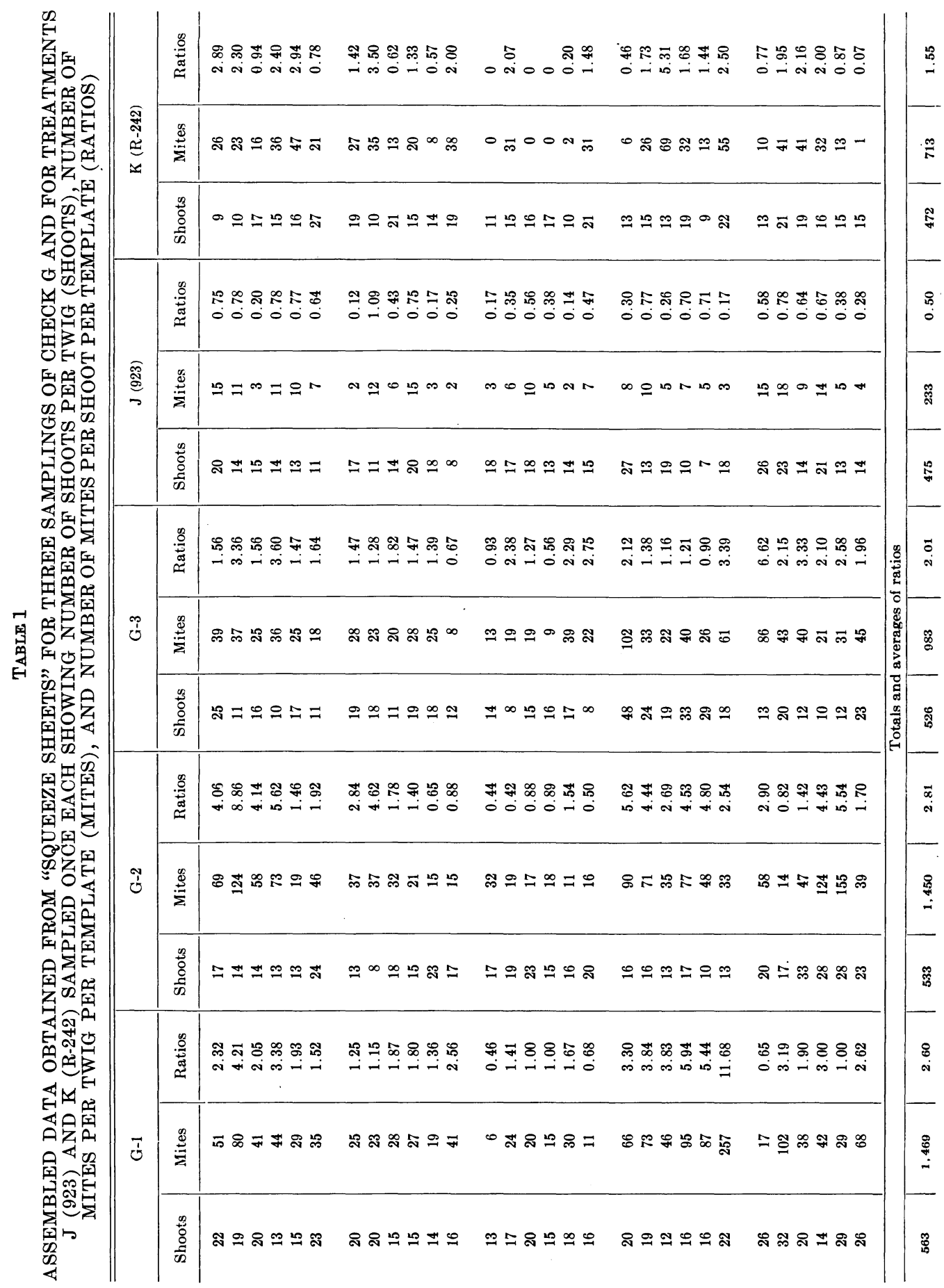


EAST

000000000000000

O(EOOOOO O'FOOOOO

000000 O O OOOOJOO

00000000000000

$0.100000 \quad 0000000$

000000000000000

$0 \bigcirc 000000000000$

000000000000000

OrAOOOBO OOOOOOOO

0000 OOOOOOOOO

00000000000000

$0 \bigcirc 00000000000$

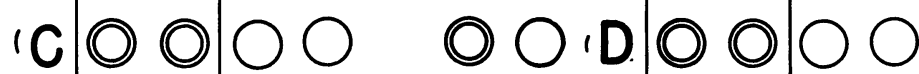

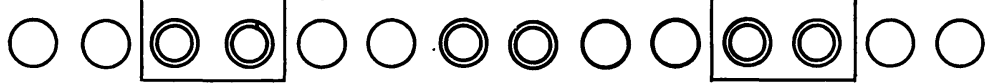

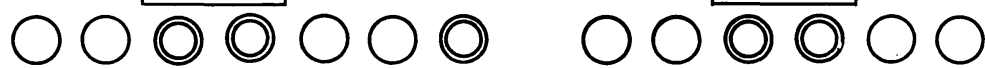

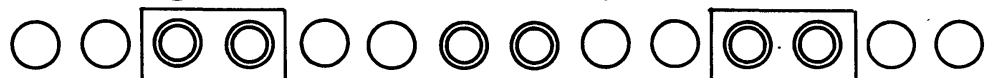

0 u $100000 \mathrm{KOO} 00$

00000000000000

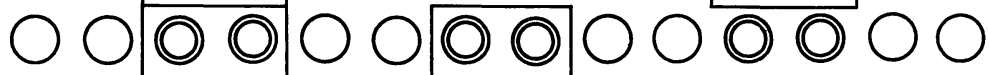

O GOOOHOOOOOOOO

OO(O) OOOOOOOOO

WEST

\section{(1) Nonpareil $\bigcirc$ Other varieties}

Fig. 1. The orchard selected for collection of data contained 15 rows of 21 trees each. The trees were fifteen years old and and of fairly uniform growth within varieties. 


\section{ANALYSIS OF TEST TRIALS}

Plots A to $\mathrm{H}$ consisted of six trees (fig. 1). Five of the six trees were selected at random and the sampling procedure described above was applied. This was repeated until three sets of data were obtained for each plot.

Check plots. The effectiveness of various methods of controlling insects, fungi, and bacteria is usually studied by laying out a replicated field trial

TABLE 2

MEANS, VARIANCES, AND M-VALUES FOR NUMBER OF

MITES PER SHOOT PER TEMPLATE FOR EACH OF THREE SAMPLINGS IN FOUR CHECK PLOTS*

\begin{tabular}{|c|c|c|c|c|}
\hline Plot & Sampling & Mean & $\begin{array}{l}\text { Over-a.l } \\
\text { variance }\end{array}$ & $\mathbf{M}$ \\
\hline \multirow{3}{*}{$\mathrm{E}$} & 1 & 4.05 & 6.88 & 11.24 \\
\hline & 2 & 3.31 & 2.28 & 8.16 \\
\hline & 3 & 4.11 & 7.58 & 13.20 \\
\hline \multirow{3}{*}{$\mathrm{F}$} & 1 & 4.07 & 3.64 & 4.67 \\
\hline & 2 & 3.72 & 6.82 & 11.68 \\
\hline & 3 & 4.19 & 11.17 & 25.16 \\
\hline \multirow{3}{*}{ G } & 1 & 2.60 & 4.83 & 24.92 \\
\hline & 2 & 2.81 & 4.33 & 13.15 \\
\hline & 3 & 2.01 & 1.43 & 10.34 \\
\hline \multirow{3}{*}{$\mathrm{H}$} & 1 & 2.12 & 2.62 & 8.20 \\
\hline & 2 & 2.17 & 3.63 & 21.64 \\
\hline & 3 & 1.86 & 1.98 & 13.38 \\
\hline
\end{tabular}

${ }^{*}$ For $\mathrm{k}=5$ and $c_{1}=0.96$ the value of $\mathrm{M}$ for the 5 per cent level of significance is 10.24 and the value for the 1 per cent level is 14.30 .

which is then analyzed by analysis of variance. If such analyses go smoothly, without difficulty in interpretation, the variances should be homogeneous throughout the experiment and independent of the level of infestation.

To test for homogeneity of variance we used $\mathrm{M}$ as defined and tabulated by Catherine M. Thompson and Maxine Merrington (1946). The M-values and means for repeated samplings of the check plots are given in table 2. High values of $\mathrm{M}$ mean nonhomogeneous variances and tend to invalidate usual analysis of variance techniques.

Since a number of the observed M's exceed the values required for significance in the tables of $M$ distribution, it is evident that some trees are more variable than others. However, the defection is not so serious as to prevent the analysis of the samplings from each check by a usual analysis of variance with some modification of the F-levels of significance (Cochran and Cox, 1950). If this somewhat rough procedure is followed, differences between 
trees are found to be significant at the 1 per cent level for plots $\mathrm{G}$ and $\mathrm{F}$ and very nearly so for plot $\mathrm{H}$. Tree differences are almost significant at the 5 per cent level for plot $\mathbf{E}$.

The means of replicate samplings are not significantly different within any of the four check plots.

On the same basis, the means and variances of plots $\mathrm{G}$ and $\mathrm{H}$ are not significantly different and the same is true of plots $\mathrm{E}$ and F. However, the mean and variances for plots $\mathrm{G}$ and $\mathrm{H}$ combined are different from those for plots $\mathrm{E}$ and $\mathrm{F}$ combined. This indicates that the level of infestation persisted over a considerable part of the orchard but not over the entire orchard. The preliminary visual appraisal of the orchard suggested that samplings from opposite ends of the rows should differ.

The facts observed so far indicate that taking a tree as a unit in a field experiment of this kind does not seem sufficient. However, four or five trees taken as a basic unit in a limited experiment that does not cover too diverse conditions should give results acceptable for use with $t$-tests based on the assumption of unequal variances.

Treated plots. The data corresponding to table 2 are given for treated plots in table 3. The last three treatments, I (K-6451), J (923), and K (R-242), bear out the previous statement that such data can be analyzed by the usual $t$-test with unequal variances. The values of $\mathrm{M}$ are moderate, indicating sufficiently uniform variances from tree to tree. Further, the trees within plots do not differ significantly in level of infestation (the F-values are 2.23, 2.18, and 1.83 when 2.76 is required for significance at the 5 per cent level). Since the trees do not differ in either means or variances, it would be possible to use twigs as fundamental units in testing the significance of the difference between these particular treatments.

The M-values for all samplings for plots A, B (TEPP) and D (TEPPS) are moderate, indicating fairly uniform variances. The very high M-values for plot C (TEPPS) might be explained by occasional spray misses. Why they should occur only in this plot is hard to say.

The means of the different samplings within plots do not show much evidence of being significantly different although the difference between the means for the first and third samplings of plot $\mathrm{D}$ approaches significance.

In testing for significance of differences between trees in the same way as before, which is certainly justified for plots A, B, and D but somewhat questionable for plot $\mathrm{C}$, we find that the treatments have smoothed out the differences between trees, leaving a fairly uniform infestation of mites. This means that twigs from these trees could also be used as fundamental units in testing differences between treatments if desired. Furthermore, plots $\mathrm{C}$ and $\mathrm{D}$ are not different but the differences between internal variances approach significance; plots A and B are significantly different in their means and approach significance in their differences between variances. This lack of correspondence in the behavior of treated plots to untreated plots may indicate lack of uniformity in spraying or, of course, residual effects of environment that persist even though a uniform spray job has been done. If this latter alternative is true, only comparatively small areas of an orchard would be suitable for testing critically small differences between treatments. 
Discussion of analysis. In an undisturbed environment (unsprayed) the mean level of infestation as determined for plots of six trees may persist over a considerable area. The populations of mites may vary significantly from tree to tree in level of infestation and in variability. The same may be true from plot to plot. However, this difference in variability may not be any greater than that encountered in field trials with yields and other char-

TABLE 3

MEANS, VARIANCES, AND M-VALUES FOR NUMBER OF MITES PER SHOOT PER TEMPLATE FOR SEVEN PLOTS, WITH FOUR PLOTS (A, B, C, D) SAMPLED THREE TIMES EACH*

\begin{tabular}{|c|c|c|c|c|}
\hline Plot and treatment & Sampling & Mean & $\begin{array}{l}\text { Over-all } \\
\text { variance }\end{array}$ & $\mathbf{M}$ \\
\hline \multirow{3}{*}{ A (TEPP) } & 1 & 3.69 & 7.43 & 8.46 \\
\hline & 2 & 2.90 & 1.83 & 5.65 \\
\hline & 3 & 3.02 & 3.71 & 15.58 \\
\hline \multirow{3}{*}{ B (TEPP) } & 1 & 2.55 & 2.61 & 5.55 \\
\hline & 2 & 2.12 & 1.63 & 3.32 \\
\hline & 3 & 2.50 & 3.02 & 11.64 \\
\hline \multirow{3}{*}{ C (TEPPS) } & 1 & 0.98 & 0.76 & 23.62 \\
\hline & 2 & 0.66 & 0.53 & 22.00 \\
\hline & 3 & 0.63 & 1.23 & 50.23 \\
\hline \multirow{3}{*}{ D (TEPPS) } & 1 & 1.17 & 2.14 & 9.83 \\
\hline & 2 & 0.84 & 1.32 & 1.99 \\
\hline & 3 & 0.53 & 0.40 & 9.40 \\
\hline I (K-6451) & 1 & 0.55 & 0.17 & 7.28 \\
\hline $\mathbf{J}(923)$ & 1 & 0.50 & 0.07 & 4.49 \\
\hline $\mathrm{K}$ (R-242) & 1 & 1.55 & 1.43 & 3.32 \\
\hline
\end{tabular}

${ }^{*} \mathrm{M}$ at 5 per cent level is $\mathbf{1 0 . 2 4}$ and at 1 per cent level 14.30 .

acteristics. The mean level of infestation as estimated by repeated samplings behaves as expected under the usual assumptions of random sampling. Areas widely separated geographically may have quite different levels of infestation.

If sprays are applied, several interesting things may happen. The differences between trees and between geographical locations may be obliterated and the variabilities for individual trees made uniform so that standard errors of experiments may be determined from the variabilities of the samples from the individual trees for each treatment. That improved experimental technique may remove plant and geographical differences has been suggested by R. E. Baker and G. A. Baker (1950) in their studies of the resistance of 
strawberries to Verticillium wilt. It is found that as the level of infestation changes markedly so does the variance of the experiment. Thus, in comparing two treatments it will in general be necessary to use a $t$-test based on the assumption of unequal variances.

On the other hand, spraying may increase tree-to-tree variabilities in some plots and not in others. Also spraying may increase plot-to-plot variabilities and introduce significant differences between levels of infestation of neighboring plots even though no such differences exist in the undisturbed situation.

It seems clear that the proposed method can be used with confidence to distinguish critically between spray materials that are of such a nature that they can be applied uniformly.

Illustrative computations. As an example of just how an analysis for the significance of differences between treatments is carried out in practice let us consider the treatments with 923 and R-242 which occur together near one end of the orchard and close to checks $G$ and $H$. Our previous examination (table 2) has shown that the checks $\mathrm{G}$ and $\mathrm{H}$ do not differ significantly and, hence, it seems reasonable to assume that the treatments with 923 and R-242 were applied to populations of mites that were originally almost uniform (except for local variation). The M-values from table 3 indicate that the samplings from the trees in each of the treated plots were sufficiently equally variable. Additional analyses given in the text show that the means of the ratios, mites per shoot per template, do not vary from tree to tree. Under these conditions, it is proper to regard the 30 ratios for treatment 923 as a random sample from a population with unknown mean and variance. Also, the 30 ratios for the plot treated with $\mathrm{R}-242$ can be regarded as a random sample from another population with unknown mean and variance. For the treatment with 923 the estimate of the mean ratio is 0.5013 and the estimate of the variance is 0.0680 . For the treatment with R-242 the estimate of the mean ratio is 1.5460 and the estimate of the variance is 1.4298 . The final level of infestation is the thing of primary interest. The significance of the difference in levels of residual infestation can be tested by considering "Student's"

$$
t=\frac{1.5460-0.5013}{\sqrt{\frac{1.4298}{30}+\frac{0.0680}{30}}}
$$

where the numerator is the difference between means and the quantity under the square root sign is the variance of the difference between the means. In this case $t$ has the value 4.68 with at least 29 degrees of freedom as indicated by Welch (1938). The probability of as great or. greater difference between means occurring by chance is less than 0.01 and thus in terms of general biological experimentation the difference between those two treatments under these particular conditions would be judged to be highly significant.

The number of ratios need not be the same in both cases and the number of trees sampled for all treatments need not be the same. However, it would be wise to keep the numbers of ratios and trees the same for every treatment. If 
wider areas and different varieties are involved, a similar analysis can be carried through but with less assurance as to the actual probability level applicable in any case.

The statistical analyses indicated as being adequate by this study are very flexible. All that is necessary to test the relative efficiency of two treatments for a given set of conditions would be to select two plots of six or more uniform trees of a single variety carrying a fairly uniform infestation of mites. Then sample the trees after treatment as described, recording the data separately for each twig by trees. Thirty twigs from each plot should be sufficient unless the variation is unusual or the distinctions to be made are very critical.

The analysis is also applicable when a comparison of samples from replicated plots with a single sample from another plot is desirable. For example, it may be desirable to compare the treatment with R-242 with the six samples drawn from replicates $G$ and $H$. From plots $G$ and $H$ the average number of mites per shoot per template in the undisturbed population is 2.2616 (average of 180 ratios). The over-all variance including variations between trees, between replications, between samples of the same trees, and between the two plots is 3.1597 . Thus, to test whether R-242 is better than the control we compute

$$
t=\frac{1.5460-2.2616}{\sqrt{\frac{1.4298}{30}+\frac{3.1597}{180}}}=\frac{-0.7156}{0.2554}=-2.80
$$

The number of degrees of freedom for $t$ is given by Welch (1938)

$$
f=\frac{\left(f_{1} \theta+f_{2}\right)^{2}}{\left(f_{1} \theta^{2}+f_{2}\right)}
$$

where $f_{1}$ is the number of items in the first sample minus one, $f_{2}$ is the number of items in the second sample minus one, and $\theta$ can be estimated as the ratio of the variance of the first sample to the variance of the second sample. Thus, in this case, $\theta=0.45$

$$
f=\frac{(0.45)(29)+(179)^{2}}{(29)(0.45)^{2}+179}=199
$$

Hence, the probability of getting a difference as great or greater than the observed value of 2.80 from "Student's" $t$-table is again less than 0.01 and is judged to be highly significant. When the smallest number of degrees of freedom is as high as 30 , to simply use the smallest number of degrees of freedom in entering "Student's" table of $t$-values usually is sufficient.

The treatment with material 923 caused a reduction of 78 per cent in the level of infestation while the treatment with material $\mathrm{R}-242$ caused a reduction of 32 per cent under the conditions of the trials.

It may be that a spray material reduces a mite population to a level more or less independent of the original level of infestation. This is more or less borne out by the removal of tree effects on sprayed plots. If this is true, it might be valid to compare the residual levels of infestation for treatments with K-6451 and 923 even though they are somewhat separated and the 
original levels of infestation were undoubtedly different. In this case sprays should be compared on the basis of their residual levels of infestation and not on the basis of percentage reduction in level of infestation. Also, comparison of sprays would be largely freed of initial conditions and could be made on the basis of final results alone. This point needs further study. In any event, we could properly compare the residual levels of infestation for treatments K-6451 and 923. The interpretation would not be entirely clear, however, because of differences in initial conditions, and the lack of knowledge of the essential behavior of spray materials; that is, whether the sprays cause a percentage decrease or permit only a certain residual population to exist.

To make such a test we need to compute

$$
t=\frac{0.5497-0.5013}{\sqrt{\frac{0.1733}{30}+\frac{0.0680}{30}}}=\frac{0.0484}{0.0890}=0.54
$$

Hence there is no significant difference between the residual levels of infestation for the treatments with K-6451 and 923.

\section{SUMMARY}

A method for estimating relative densities of brown almond mite populations on mature almond trees has been devised and applied in uniformityand treatment-trials. The chief advantages of the method are: 1) that it is not appreciably affected by diurnal changes in the distribution of mites on leaves and wood as related to changes of temperature and sunlight; and 2) the large numbers of mites recovered from whole twigs are as easily dealt with in random sampling as are the few mites encountered on samples of leaves picked one at a time. The ecological basis and the mechanics of the sampling process are described. The unit values (ratios) obtained are actually samples of samples; that is, mites per twig (expressed as the average number of mites for each shoot borne on the twig) and mites per template. Active mites are beaten from twig samples onto sheets of paper and fixed into position by crushing. A sample of the unevenly dispersed blots (or dried, crushed mites) is made by using a template. Each value finally obtained-mites per shoot per template-is useful for comparing population densities in relative terms.

The sampling process was evolved by trial and error and is known to have many sources of unavoidable error. The analyses given represent an attempt to isolate the major sources of variation and to prescribe the simplest statistical method applicable to the type and quantity of data furnished by this arbitrarily fixed procedure. The results obtained in these trials are appropriate for statistical consideration because, in this set of experiments, none of the treatments produced decisive or outstanding results in control.

Tests for homogeneity of variances were applied to these data from uniformity trials in order to determine how variance analysis can be applied for routine decisions as to the significance of differences between treatments. Since the variance of samples depends on the level of infestations, $t$-tests based on the assumption of unequal variance must be used to test the significance 
of the differences between treatments. The design indicated as being adequate for testing relative efficiency of treatments requires four to five similar trees of one variety carrying a fairly uniform infestation of mites. Thirty individual twigs from each plot are believed to be sufficient unless variations are unusual or the distinctions to be made are very critical. Several illustrative calculations are given.

\section{LITERATURE CITED}

BAKer, R. E., and G. A. BAKer

1950. Experimental designs for studying resistance of strawberry varieties to Verticillium wilt. Phytopath. 40(5):477-82.

Cochran, W. G., and G. M. Cox

1950. Experimental designs. 454 pp. John Wiley and Sons, Inc., New York.

Thompson, Catherine M., and Maxine Merrington

1946. Tables for testing the homogeneity of a set of estimated variances. [Prefatory note by H. O. Hartley and E. S. Pearson.] Biometrika 33(4) :296-301.

WELCH, B. L.

1938. Significance of the difference between two means when the population variances are unequal. Biometrika 29 (Parts I and II) :350-62.

SuMmers, Francis M.

1950. New data on brown almond mite. Almond Facts 14(2):8.

In order that the information in our publications may be more intelligible, it is sometimes necessary to use trade names of products or equipment rather than complicated descriptive or chemical identifications. In so doing, it is unavoidable in some cases that similar products which are on the market under other trade names may not be cited. No endorsement of named products is intended nor is criticism implied of similar products which are not mentioned. 
The journal Hilgardia is published at irregular intervals, in volumes of about 600 pages. The number of issues per volume varies.

Subscriptions are not sold. The periodical is sent as published only to libraries, or to institutions in foreign countries having publications to offer in exchange.

You may obtain a single copy of any issue free, as long as the supply lasts; please request by volume and issue number from:

\section{Publications Office \\ College of Agriculture \\ Berkeley 4, California}

The limit to nonresidents of California is 10 separate issues on a single order. A list of the issues still available will be sent on request. 